B), and a control group of 74 with a CTX MIC of $<0.125 \mu \mathrm{g} / \mathrm{ml}$ (group $\mathrm{C}$ ), were included. All isolates were characterised using antibiograms, conventional penA mosaic gene PCR, and genotyping by NG-multi-locus variable-number tandem repeat analysis (MLVA). PenA mosaic positive isolates and a strict selection of the remaining isolates were further characterised by NG-multilocus sequence typing (MAST) and sequencing of ESC resistance determinants (penA, mtrR, and porB1b).

Results The majority of the isolates in group A ( $n=47 ; 64 \%$ ) but only $11 \%(n=6)$ of the isolates in group B contained a penA mosaic allele. No penA mosaic-containing isolate was identified in group C (see Abstract O1-S03.03 figure 1). All the 53 penA mosaic isolates had an identical penA sequence (type XXXIV) and were assigned to the same MLVA cluster, which additionally included three isolates that were susceptible to CTX $(\mathrm{MIC}<0.125 \mu \mathrm{g} / \mathrm{ml})$. Within this MLVA cluster, $46(87 \%)$ of the penA mosaic isolates were assigned NG-MAST ST1407, and the remaining 7 isolates had closely related STs. All these penA mosaic isolates contained a mtrR promoter deletion and in 52/53 isolates the porB1b alterations $\mathrm{G} 101 \mathrm{~K}$ and $\mathrm{A} 102 \mathrm{~N}$ were found. Decreased susceptibility to cefixime and ceftriaxone $(\mathrm{MIC} \geq 0.016 \mu \mathrm{g} / \mathrm{ml})$ was found in 50/53 and 44/53 isolates, respectively. The mosaic penA MLVA cluster, containing ST1407 (87\%) and closely related STs (13\%), represented Dutch homosexual men (66\%), patients with frequent chlamydia co-infection (32\%), and commercial sex workers (7\%).

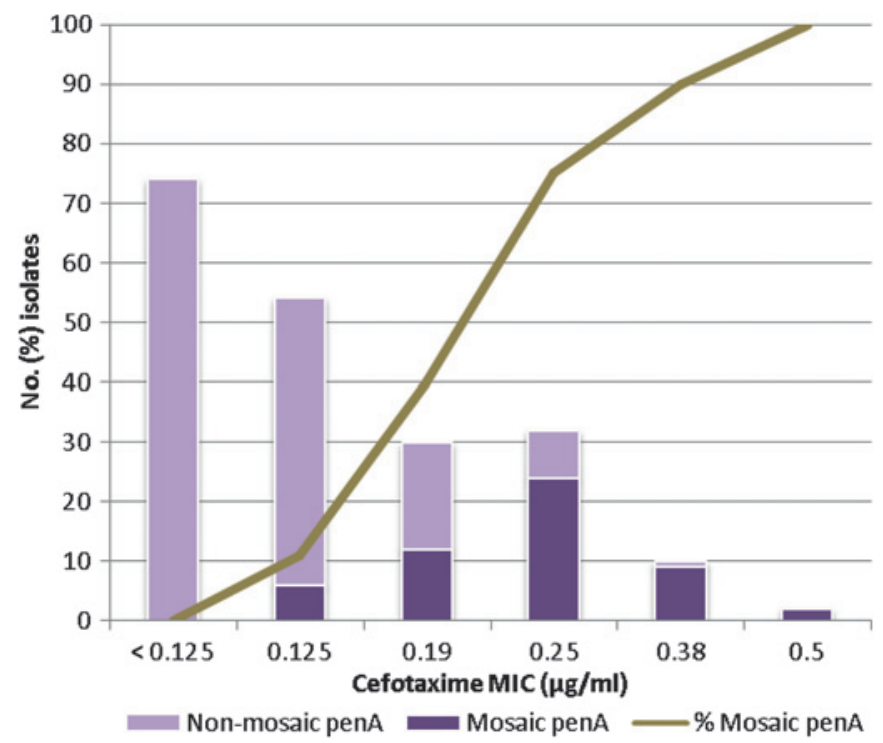

Abstract 01-S03.03 Figure 1 GGD.

Conclusions A strong correlation was found between the decreased ESC susceptibility and a NG penA mosaic strain (ST1407) that was highly prevalent among visitors of the STI clinic Amsterdam. This strain was identified in many other countries. The rapid spread of this NG strain might be facilitated by high-risk sexual behaviour and should be monitored closely to identify potential treatment failure.

\section{1-S03.04 CORE GROUPS, ANTIMICROBIAL RESISTANCE AND REBOUND IN GONORRHOEA}

doi:10.1136/sextrans-2011-050109.16

${ }^{1} \mathrm{C}$ Chan, ${ }^{2} \mathrm{D}$ Fisman, ${ }^{1} \mathrm{C}$ McCabe. ${ }^{1}$ University of Toronto, Toronto, Canada; ${ }^{2}$ University of Toronto, Faculty of Medicine, Toronto, Canada

Background Neisseria gonorrhoeae (NG) is a major cause of sexually transmitted infection worldwide. Surveillance data from North America suggest that incidence has increased in recent years, after initially falling in the face of intensified control efforts, as anti- microbial resistance in NG has increased. We evaluated the likely mechanisms behind such rebound using simple compartmental models, and explored the implications of such rebound for disease control practice.

Methods We evaluated the impact of risk-focussed treatment strategies on long-term gonorrhoea trends using risk-structured susceptible-infectious-susceptible" (SIS) compartmental models that included and excluded the possibility of antibiotic resistance in gonorrhoea transmission and control. We also examined optimal treatment strategies to minimise gonorrhoea rates when more than one antibiotic is available.

Results Model projections, consistent with previous work, showed that when antibiotic resistance is not possible, strategies that focus on treatment of highest risk individuals (the so-called "core group"), result in collapse of gonorrhoea transmission see Abstract O1-S03.04 figure 1 . In contrast, in the presence of antimicrobial resistance, a focus on the core group causes rebound in incidence, with maximal dissemination of antibiotic resistance. When two antibiotics are available for treatment, we found that random assignment of treatment was most effective at delaying rebound in overall rates in the population, while the current strategy, which is to switch firstline treatment when a threshold level of resistance is reached, produced the quickest rebound.

Conclusions While previous models have shown that the targeted treatment of core-group individuals is the most effective at lowering rates of gonorrhoea, our model suggests that core group-focused treatment strategies efficiently disseminate antimicrobial resistant strains of NG, with rebound in gonorrhoea rates. This paradox poses a great dilemma to the control and prevention of gonorrhoea, especially when development of new antibiotic classes has lagged in recent years and vaccine development for gonorrhoea still faces many challenges. Our study highlighted the need for focus on nonantimicrobial strategies for the prevention and control of gonorrhoea.

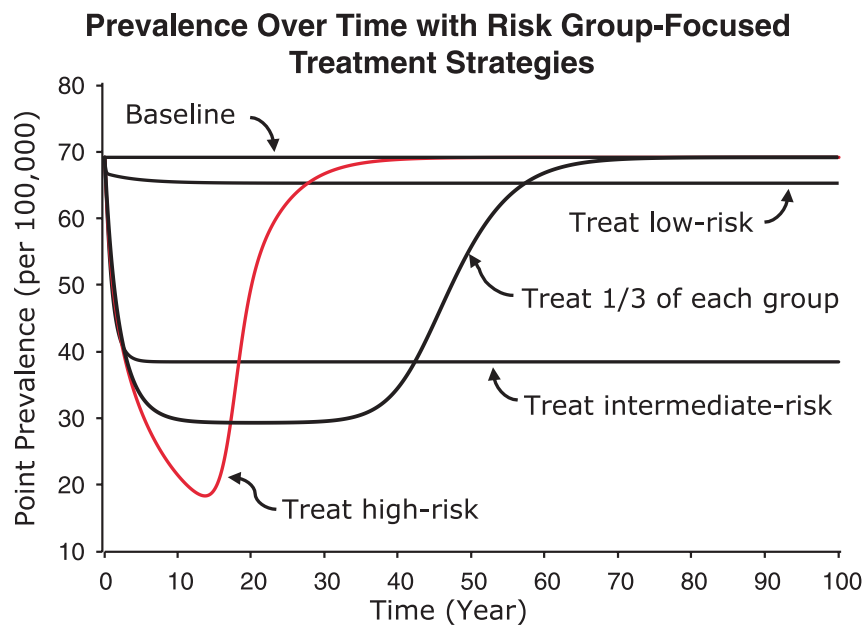

Abstract 01-S03.04 Figure 1 Prevalence over time with risk groupfocused treatment strategies.

\section{1-S03.05 COMMUNITY-ACQUIRED METHICILLIN-RESISTANT AND SUSCEPTIBLE STAPHYLOCOCCUS AUREUS AMONG MEN WHO HAVE SEX WITH MEN}

doi:10.1136/sextrans-2011-050109.17

H De Vries, I Joore, M van Rooijen, M Schim van der Loeff, A van Dam, H De Vries. GGD Amsterdam, Amsterdam, Netherlands

Background Community Acquired Methicillin Resistant Staphylococcus aureus (CA-MRSA) has been found more often among men who have sex with men (MSM) in some studies (USA). This study assesses the prevalence and sexual risk factors for CA-MRSA 